\title{
ITERATIVE LOCALISATION IN WIRELESS AD HOC SENSOR NETWORKS: ONE-DIMENSIONAL CASE
}

\author{
Aditya Kamik* and Anurag Kumar ${ }^{\dagger}$ \\ Department of Electrical Communication Engineering, \\ Indian Institute of Science, Bangalore, India 560012
}

\begin{abstract}
This paper discusses a simple method of localisation in sensor networks in which a sensor with unknown location is localised to a disk of radius equal to the transmission range centered at a beacon if the sensor under consideration can receive a transmission from the beacon. This is a reliable and extremely easy-to-implement technique since it assumes only the basic communication capability. The real advantage, however, is that once localised, a sensor can aid the other sensors to localise. This way by collaboration sensors can learn and improve their localisation regions iteratively. We analyse this iterative scheme and construct a distributed algorithm for utilising it in sensor networks.
\end{abstract}

\section{INTRODUCTION}

Spatial or location information is of intrinsic interest in sensor networks; for example, it is essential in applications that involve data combining and estimation. However, such information can neither be pre-configured in sensors owing to their ad hoc and possibly randorn deployment nor can it be centrally disseminated to sensors because of the absence of a centralised coordinator. Further, because of cost and power constraints only a few sensors can be equipped with Global Positioning System (GPS) receivers. Thus, it is imperative that sensors infer their locations autonomously using only a few sensors which have knowledge of their location either through mounted GPS receivers or a prioni placement with preset coordinates

This is not a new problem; locating objects in two dimensions (e.g., surface of the earth) or three dimensions (e.g., space) from the knowledge of locations of some distinguished nodes, called beacons, has been the central problem in navigation. Location of a node can be known from its distances and/or angles to beacons. For example, on the plane, if distances to at least three beacons are known

\footnotetext{
*Currently with STCS, TIFR. Mumbai. Research supported by a fellowship from the IBM India Research Lab.

${ }^{\dagger}$ Research supported by a grant from the Indo-French Centre for the Promotion of Advanced Research (IFCPAR) (Project No. 2900-IT)
}

from a node, then its position can be fixed ${ }^{1}$. The distance measurements in this context are generally referred to as ranging. What distinguishes the localisation problem in sensor networks from the navigation problem is the following. Due to spatial expanse of a sensor network not every sensor will have the required number of beacons for ranging; to be cost effective, fewer beacons are desired. In addition, the traditional ranging methods based on received signal strength (RSSI), time of arrival (TOA), angle of arrival (AOA), time difference of arrival (TDOA), etc. ([3]) have several shortcomings from point of view of the sensor networks. RSSI is usually very unpredictable since the received signal power is a complex function of the propagation environment. Hence, radios in sensors will need to be well calibrated otherwise sensors may exhibit significant variation in power to distance mapping. TOA using acoustic ranging requires an additional ultrasound source. TOA and RSSI are affected by measurement as well as non-line of sight errors. TDOA is not very practical for a distributed implementation. AOA sensing will require either an antenna array or several ultrasound receivers.

This motivates us to consider a particularly simple method of localisation which we call the in-range method (IR). The basic premise of IR is that a transmission at a given power can be decoded only up to a maximum distance, called its transmission range. IR then simply localises a node with unknown location to a disk of radius equal to the range centered at a beacon if the node under consideration can successfully decode a transmission from the beacon. Figure 1 shows an example of IR localisation. Let $R$ denote the range and $D(x, r)$ a disk of radius $r$ centered at $x$. In Figure 1 , $B_{i}$ is a beacon with location $v_{i}$ for $i=1,2,3$. The solid circles indicate disks $D\left(v_{i}, R\right), i=1,2,3$. The dotted circles correspond to exact ranging. Thus, $S$ is localised to $X=D\left(v_{1}, r_{1}\right) \cap D\left(v_{2}, r_{2}\right) \cap D\left(v_{3}, r_{3}\right)$, i.e., the region of intersection of three solid circles. We will call $X$ the localisation set (or region) of sensor $S$.

Though crude as compared to other ranging methods, IR is a reliable and easy to implement technique which as- ,

\footnotetext{
'Since no measurements are exact in practice, the location can only be
} estimated. 

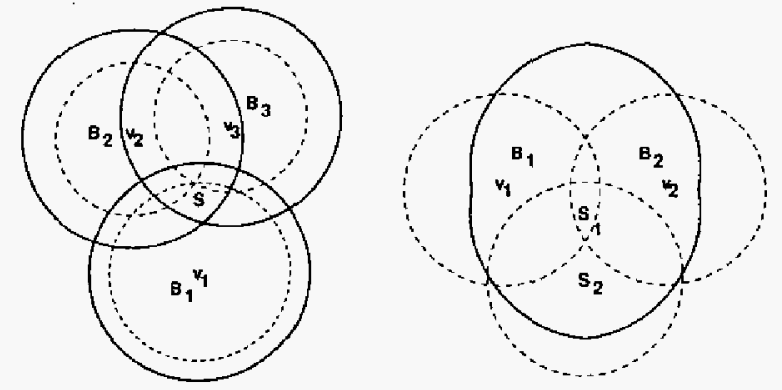

Fig. 1. Localisation with IR in the plane.

sumes only a basic communication capability. The advantage of IR, however, is that once localised, a sensor can aid other sensors in localising and/or in reducing the lo. calisation sets of some other previously localised sensors. Figure 1 shows such a scenario. $S_{1}$ and $S_{2}$ are two sensors with unknown locations. $S_{1}$ is in the range of beacons $B_{1}$ and $B_{2} ; B_{i}$ located at $v_{i}$. Therefore, it gets localised to the region of intersection of $D\left(v_{i}, R\right), i=1,2$ shown by dotted circles with centers $v_{1}$ and $v_{2} . S_{2}$, though not in the range of either $B_{1}$ or $B_{2}$, is in the range of $S_{1}$; the dotted circle centered at $S_{1}$ is $D\left(v_{s_{1}}, R\right)$ where $v_{s_{1}}$ denotes the location of $S_{1}$. Therefore, $S_{2}$ gets localised to the region bounded by the solid curve shown in Figure 1. This way sensors can leam and improve their localisation sets iteratively. The objective of this work is to investigate this iterative localisation process when sensors and beacons are randomly placed and to construct a distributed algorithm for utilising this scheme in sensor networks. In this paper we confine our discussion to one dimensional sensor networks. Extension to planar sensor networks has been done in [4] and will be discussed in an extended version of this paper.

This paper is organised as follows. Related work is reviewed in Section 2. In Section 3 we describe the general IR scheme and analyse it in the one dimensional case in Section 4; for brevity we omit the formal proofs which can found in [4]. Results are discussed in Section 5. A distributed algorithm based on this scheme is presented in Section 6. We conclude in Section 7.

\section{RELATED WORK}

The localisation scheme in [5] uses RSSI based distance estimates to beacons whereas in [1] it is based on TOA with acoustic ranging and multilateration. Both these methods have limitations discussed in Section 1. The basic premise of [2] is that the number of communication hops between 2 sensors gives easy and reasonably accurate distance estimates at higher densities. Such distance estimates to many beacons are then used in a gradient descent algorithm at a sensor to minimise the location error. In its basic idea [6] is the closest to our work, i.e., a sensor with unknown location is localised to a "rectangle" centered at a beacon if the sensor can hear a "Hello" from it. However, the analysis is in a discrete setting and the localisation scheme is not iterative.

\section{ITERATIVE LOCALISATION USING IR}

Consider a randomly deployed sensor network in a geographical region $\mathcal{A}$; in this paper $\mathcal{A} \subset \mathbb{R}$. The sensors are indexed by $i \in\{1,2, \ldots, N\}$. We say that a transmission can be "decoded" by a sensor when its signal to interference ratio (SIR) exceeds a given threshold $\beta$. The transmission range is then defined as the maximum distance at which a receiver can decode a transmitter in the absence of any co-channel interference. We denote the transmission range of sensors by $R_{0}$. The sensors within $R_{0}$ from $i$ will be called its neighbours. The set of neighbours of $i$ will be denoted by $N_{i}$ and their number by $n_{i}$. By the location of sensor $i$ we mean its coordinates and denote it compactly by $v_{i}$; in this paper $v_{i}$ is just the $x$-coordinate of sensor $i$. A localisation set for a sensor $i$ is a subset of the region of deployment. Let $X_{i}(0)$ denote the initial localisation set for $i=1,2, \ldots, N$. Recall that beacons are those sensors which know their location; the others are calted ordinary sensors or simply sensors. Thus if $i$ is a sensor then $X_{i}(0)=\mathcal{A}$ else $X_{i}(0)=\left\{v_{i}\right\}$. Recall that $D(v, r)$ denotes the disk of radius $r$ centered at $v$; in one dimension disks are "intervals". 0 denotes the origin. If $G$ and $H$ are iwo sets, $G+H$ denotes the set addition, i.e., $G+H=\{g+h \mid g \in G, h \in H\}$.

The following gives the iterative IR scheme. $n$ is the iteration index. For $n \geq 0$ and $i=1,2, \ldots, N$,

$$
\begin{aligned}
& Y_{i}(n+1)=\bigcap_{k \in N_{i}}\left(X_{k}(n)+D\left(0, R_{0}\right)\right) \\
& X_{i}(n+1)=X_{i}(n) \cap Y_{i}(n+1)
\end{aligned}
$$

$X_{k}(n)$ denotes the localisation set of node $k$ in iteration $n$. Therefore, if $i$ is in the range of $k, i$ is certainly in the region $X_{k}(n)+D\left(0, R_{0}\right)$. Since this property holds for each neighbour of $i, i$ is localised to $\bigcap_{k \in N_{i}}\left(X_{k}(n)+D\left(0, R_{0}\right)\right)$. Thus, it follows that the localisation set of $i$ in the $(n+1)^{\text {th }}$ iteration is the intersection of its localisation set in the $n^{\text {th }}$ iteration and $\bigcap_{k \in N_{i}}\left(X_{k}(n)+D\left(0, R_{0}\right)\right)$.

Let $\mathcal{L}(X)$ denote a measure of set $X$; in one dimension it is the length of $X$. Define, $\chi_{i}(n):=\mathcal{L}\left(X_{i}(n)\right)$. We call $\chi_{i}(n)$ the localisation error of sensor $i$ in iteration $n$. Let $\underline{\chi}(n):=\left(\chi_{1}(n), \chi_{2}(n), \ldots, \chi_{N}(n)\right)$ and consider the vector valued process $\{\chi(n), n \geq 0\}$ which we call the localisation process. Note from (2) that for each $i, \chi_{i}(n)$ is nonincreasing with $n$. Let $A$ denote $\mathcal{L}(A)$. We say that sensor $i$ is localised by (i.e., at or before) iteration $n$ if $\chi_{i}(n)<A$. Then the perfornance measures which are of interest are 
$\bar{\chi}(n):=\frac{1}{N} \sum_{i=1}^{N} \chi_{i}(n)$ and $\nu(n):=\frac{1}{N} \sum_{i=1}^{N} 1_{\left\{\chi_{i}(n)<A\right\}}$ where, $1_{\{.\}}$denotes the indicator function. Thus, by definition $\bar{\chi}(n)$ is the average localisation error in the network at the end of $n$ iterations and $\nu(n)$ is the fraction of nodes localised by iteration $n$.

\section{ONE DIMENSIONAL LOCALISATION PROCESS}

We assume $N$ to be very large and model the random dispersion of sensors on the real line as a one dimensional Poisson point process $\Psi$ of intensity $\lambda$; Poisson points indicate locations of sensing nodes. A fraction $p$ of the nodes are beacons. In the point process model we assume that a node is a beacon with probability $p$ independent of anything else. It follows that locations of beacons and sensors form independent Poisson processes of intensity $\lambda p$ and $\lambda(1-p)$ ) respectively.

Recall that $v_{j}$ denotes the $\mathrm{x}$ coordinate of $j$. Thus, disk $D\left(v_{j}, R_{0}\right)$ extends from $v_{j}-R_{0}$ to $v_{j}+R_{0} . \chi_{j}(n)$ is the length of $X_{j}(n)$. The length of the localisation interval lying to the right of $j$ is denoted by $\Delta_{j}^{r}(n)$ while the length of the interval lying to the left of $j$ is denoted by $\Delta_{j}^{l}(n)$; hence, $\chi_{j}(n)=\Delta_{j}^{r}(n)+\Delta_{j}^{l}(n)$. If $j$ is a beacon, $\chi_{j}(n)=\Delta_{j}^{r}(n)=\Delta_{j}^{l}(n)=0, n \geq 0$. If $j$ is an ordinary sensor, $\Delta_{j}^{r}(0)=\Delta_{j}^{l}(0)=\frac{A}{2}$. In the Poisson process model, $A$ should be interpreted as a value representing the initial uncertainty of each sensor. The evolution (2) in this setting is as follows. Recall that $N_{j}$ denotes the set of neighbours of $j$. For $n \geq 1$ and $j=1,2, \ldots, N$,

$$
\begin{aligned}
u_{j}^{r}(n)= & \arg \min _{k \in N_{j}}\left(v_{k}+\Delta_{k}^{r}(n-1)\right) \\
\Delta_{j}^{r}(n)= & \min \left(\Delta_{j}^{r}(n-1),\right. \\
& \left.v_{u_{j}^{r}(n)}+\Delta_{u_{j}^{r}(n)}^{r}(n-1)+R_{0}-v_{j}\right) \\
u_{j}^{l}(n)= & \arg \max _{k \in N_{j}}\left(v_{k}-\Delta_{k}^{l}(n-1)\right) \\
\Delta_{j}^{l}(n)= & \max \left(\Delta_{j}^{l}(n-1),\right. \\
& \left.v_{j}-v_{u_{j}^{l}(n)}+\Delta_{u_{j}^{l}(n)}^{l}(n-1)+R_{0}\right) \\
\chi_{j}(n)= & \Delta_{j}^{r}(n)+\Delta_{j}^{l}(n)
\end{aligned}
$$

If $N_{j}$ is empty, then by convention the minimum over an empty set is taken to be $\infty$ and we define the location of $u_{j}^{r}(n+1)$ to be $\infty$. Similarly for $u_{j}^{l}(n+1)$.

To understand the iterative process given by (3), let us first consider $n=1$. Assume that $j$ is a sensor. Since $\Delta_{k}^{r}(0)=0$ if $k$ is a beacon and $\frac{A}{2}$ otherwise, it follows that, $X_{j}(1)$ is decided only by the beacons in its range. Further, $X_{j}(1)$ will be determined by the leftmost and the rightmost beacon in the range of $j$; the leftmost beacon will determine $\Delta_{j}^{r}(1)$ and the rightmost beacon will determine $\Delta_{j}^{l}(1)$. Step (3) locates the leftmost beacon; it is denoted by $u_{j}^{r}(\mathrm{I})$. Then it is easy to see that $\Delta_{j}^{r}(1)=v_{u_{j}^{r}(1)}+R_{0}-v_{j}$ since $R_{0}$ is

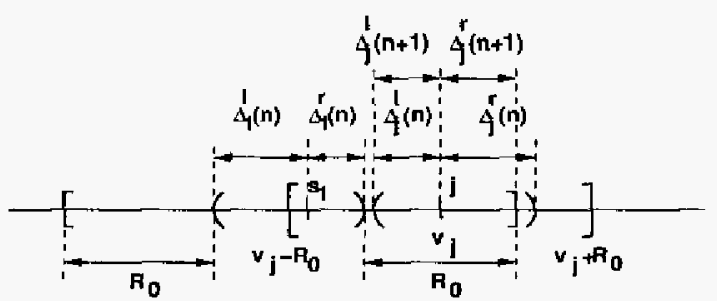

Fig. 2. An example of the propagation of $\Delta_{j}^{r}(n)$ and $\Delta_{j}^{l}(n)$.

the range and $v_{j}$ is $j$ s location. Similarly $u_{j}^{l}(1)$ denotes the rightmost beacon so that $\Delta_{j}^{j}(1)=v_{j}-v_{u_{j}^{l}(1)}+R_{0}$.

Now for $n \geq 2$, consider an example shown in Figure 2 . Assume that $j$ has only one neighbour denoted by $s_{1}$ with location $v_{1}$. Suppose that $s_{1}$ is a sensor. Further, $n-1$ iterations are over; $j$ has been localised to $\left[v_{j}-\Delta_{j}^{l}(n-\right.$ 1), $\left.v_{j}+\Delta_{j}^{r}(n-1)\right]$ and $s_{1}$ to $\left[v_{1}-\Delta_{1}^{t}(n-1), v_{1}+\Delta_{1}^{r}(n-\right.$ 1)]. Since $j$ lies in the transmission range of $s_{1}$ by virtue of this single constraint, $j$ must lie within $\left[v_{1}-\Delta_{1}^{l}(n-\right.$ 1) $-R_{0}, v_{1}+\Delta_{1}^{r}(n-1)+R_{0}$ ] shown by the small square brackets. The length of the "right" side of this interval is $v_{1}+\Delta_{1}^{r}(n-1)+R_{0}-v_{j}$. It follows that $\Delta_{j}^{r}(n)$ will be the minimum of $\Delta_{j}^{r}(n-1)$ and $v_{1}+\Delta_{1}^{r}(n-1)+R_{0}-v_{j}$. Similar analysis applies to $\Delta_{j}^{l}(n)$. Equations (3) and (4) simply extend this logic to a general case.

Though (3) and (4) are much simplified compared to (2), the process $\underline{\chi}(n)$ is still not amenable to analysis. We now work with a typical point of the Poisson process, called the tagged node (denoted by $o$ ) and study the "coordinate" process of $\underline{\chi}(n)$, i.e., $\left\{\chi_{0}(n), n \geq 0\right\}$, the sequence of localisation errors of the tagged node. The performance measures discussed in Section 3 can be obtained at the tagged node as, $\bar{\chi}(n)=E_{\chi_{0}}(n), \nu(n)=P\left(\chi_{o}(n)<A\right)$.

\subsection{The Coordinate Process, $\left\{\chi_{0}(n), n \geq 0\right\}$}

If $o$ is a sensor then $X_{o}(0)=\mathcal{A}$ else $X_{o}(0)=\{0\}$. The earlier discussion has shown that if $o$ is a sensor $X_{o}(1)$ is decided only by the beacons in the range of $o$. It is thus possible to explicitly characterise the distribution of $\chi_{0}(1)$ ([4]). However, for further analysis we will work with a simpler process, $\left\{\Delta_{0}^{r}(n), n \geq 0\right\}$. Note that, $\Delta_{o}^{r}(1)$ and $\Delta_{o}^{l}(1)$ are identically distributed though not independent. By symmetry, this property holds for $n \geq 2$.

Proposition 4.1 ([4]) Ifo is a sensor, the probability distribution of $\Delta_{o}^{r}(1)$ is,

$$
P\left(\Delta_{o}^{r}(1) \leq y\right)= \begin{cases}1 & y>\frac{A}{2} \\ 1-e^{-\lambda p 2 R_{0}} & 2 R_{0}<y<\frac{A}{2} \\ 1-e^{-\lambda p y} & 0<y \leq 2 R_{0}\end{cases}
$$

with probability mass at $A / 2: P\left(\Delta_{\circ}^{r}(1)=A / 2\right)=e^{-\lambda p 2 R_{0}}$. 


\section{Corollary 4.1}

$\frac{E \Delta_{o}^{r}(1)}{(1-p)}=\frac{1-e^{-\lambda p 2 R_{0}}-\lambda p 2 R_{0} e^{-\lambda p 2 R_{0}}}{\lambda p}+\frac{A}{2} e^{-\lambda p 2 R_{0}}$

Observe from (3) that, for a given $\Delta_{o}^{r}(1), \Delta_{o}^{r}(n), n \geq 2$ is determined by the (ordinary) sensors in the range of $o$. Let $N_{o}^{s}$ denote the set of sensors in the range of $o$ and $n_{o}^{s}$ their number. Consider now the iteration (3) applied to 0 for $n \geq 2 . u_{o}^{r}(n):=\arg \min _{k \in N_{s}^{*}}\left(u_{k}+\Delta_{k}^{r}(n-1)\right)$ and

$\Delta_{o}^{r}(n)=\min \left(\Delta_{o}^{r}(n-1), v_{u_{o}^{r}(n)}+\Delta_{u_{o}^{r}(n)}^{r}(n-1)+R_{0}\right)$

Recall that if $N_{o}^{s}$ is empty, by convention the minimum over $N_{o}^{s}$ in (6) is infinite and $\Delta_{o}^{r}(n)=\Delta_{o}^{r}(n-1)$. Now a direct analysis of (6) amounts to analysing $\{\chi(n), n \geq 0\}$ since to find the probability distribution of $\bar{\Delta}_{o}^{r}(n)$, we need the joint distribution of $\Delta_{k}^{r}(n-1), k \in N_{0}^{s}$. However, an asymptotically exact approximation for $\left\{\chi_{o}(n), n \geq 2\right\}$ can be obtained as follows.

Since $o$ is a typical point of Poisson process, $n_{o}^{s}$ is Pois. son distributed with mean $\lambda(1-p) 2 R_{0}$. We denote by $o_{k}$ the $k^{t h}$ "sensor-neighbour" of $o$. For a given $n_{o}^{s}$. $v_{o_{k}}$ are independent uniformly distributed random variables in $\left[-R_{0}, R_{0}\right]$. We now index these neighbours by $i \in\left\{1, \ldots, n_{o}^{s}\right\}$ based on the order statistics of $v_{o_{k}}$, i.e., the sensor corresponding $i^{\text {th }}$ smallest value of $v_{o_{k}}$ 's is indexed $i$. Thus 1 $\left(\arg \min _{1 \leq k \leq n_{v}^{s}} v_{o_{k}}\right)$ is the "leftmost" neighbour and the rest in the increasing order towards right. Location of $i$ is denoted by $v_{i} ; f_{v_{1}}\left(x \mid n_{o}^{s}=m\right)=\frac{m}{2 R_{0}}\left(1-\frac{x+R}{2 R_{0}}\right)^{m-1}$. Now consider a sequence $\left\{\hat{\Delta}_{o}^{r}(n), n \geq 0\right\}$ such that $\hat{\Delta}_{o}^{r}(1)=$ $\Delta_{o}^{r}(1)$ and for $n \geq 2$,

$$
\hat{\Delta}_{o}^{r}(n)=\min \left(\hat{\Delta}_{o}^{r}(1), v_{1}+\hat{\Delta}_{o}^{r}(n-1)+R_{0}\right)
$$

Thus, $\left\{\Delta_{o}^{r}(n), n \geq 2\right\}$ can be generated iteratively; computation of the statistics of $\hat{\Delta}_{o}^{r}(n)$ requires only the statistics of $\Delta_{o}^{r}(1)$ and that of $\hat{\Delta}_{o}^{r}(n-1)$ computed in the previous iteration. Let $F_{\Delta_{o}^{(n)}(x)}(x)$ (respectively, $F_{\Delta_{\delta}^{r}(n)}(x)$ ) denote the cumulative probability distribution of $\Delta_{o}^{r}(n)$ (respectively, $\hat{\Delta}_{o}^{r}(n)$ ). Then the following holds.

Proposition 4.2 ([4]) For $n \geq 1$.

$$
\lim _{\lambda \rightarrow \infty}\left|F_{\Delta_{u}^{r}(n)}(x)-F_{\hat{\Delta}_{o}^{x}(n)}(x)\right|=0
$$

\subsection{The Process, $\{\nu(n), n \geq 0\}$}

Recall that $\nu(n)=P\left(\chi_{o}(n)<A\right)$, the fraction of nodes which get localised by iteration $n$. Since all the sensors need to get localised it is important to study $\{\nu(n), n \geq 0\}$.

Proposition $4.3([4]) \nu(1)=p+(1-p)\left(1-e^{-\lambda p 2 R_{0}}\right)$ and

$$
\lim _{\lambda \rightarrow \infty}\left(p+(1-p)\left(1-e^{-\lambda p n 2 R_{0}}\right)-\nu(n)\right)=0
$$

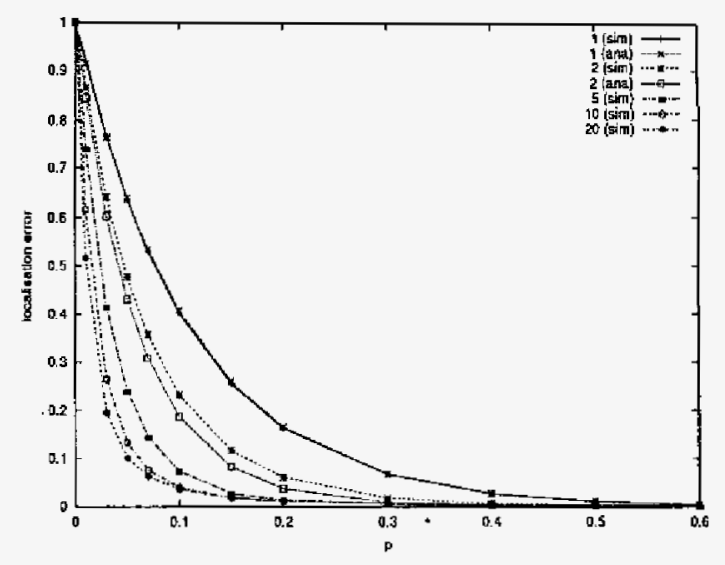

Fig. 3. Localisation error (normalised to $A$ ) vs. $p$ for $\lambda=2$ per $\mathrm{m}, R_{0}=2 \mathrm{~m}, A=200 \mathrm{~m}$. Analysis and Simulation.

\section{RESULTS AND DISCUSSION}

Figure 3 shows the variation of average localisation error (nomalised to $A$ ) with the beacon density $p$ for increasing iterations when $\lambda=2$ per $m$. $A$ is obtained as follows. In the simulation, we generate 1000 Poisson points for the random sensor placement model. The initial uncertainly for each serasor is then the expected length of this placement, i.e., $A=\frac{1000}{\lambda}$ and the initial location is its center. $E \chi_{o}(1)$ and $E \hat{\chi}_{o}(2)$ are also obtained from the previous analyses (Section 4.1). Note from Figure 3 that, in the case of $E_{\chi_{o}}(1)$ the simulation results perfectly match the analysis. For $E \chi_{0}(2)$, the analytical result gives a fairly good match even for a low value such as $\lambda=2$. For higher densities, analytical values match extremely well with those obtained by simulation ([4]). This suggests that (7) may be used to characterise the localisation errors iteratively for values of intensity of practical interest. Figure 3 also shows the benefits of the iterative scheme. When $10 \%$ sensors are beacons $(p=0.1)$, the localisation error is reduced from $40 \%$ in the first iteration to less than $5 \%$ in $20^{\text {th }}$ iteration.

Figure 4 shows the variation of $v(n)$ with $p$ when $\lambda=2$ per $m . v(1)$ and $v(2)$ are also obtained analytically (see Section 4,2). Observe that $\left(p+(1-p)\left(1-e^{-\lambda p n 2 R_{i j}}\right)\right)$ is an upper bound on $v(n)$ for $n \geq 2$. This follows from the analysis ([4]). On the other hand, for higher densities, the analytical and simulation results are in excellent match. Note from Figure 4 that with $10 \%$ beacons, starting with about $60 \%$ nodes getting localised in the first iteration, by $20^{\text {th }}$ iteration about $98 \%$ the nodes are localised whereas with $20 \%$ beacons, starting with about $80 \%$ localised nodes in the first iteration, by $20^{\text {th }}$ iteration almost all the nodes are localised and the localisation error is less than $1 \%$ (Figure 3 ).

It is important to understand that effectiveness of IR 


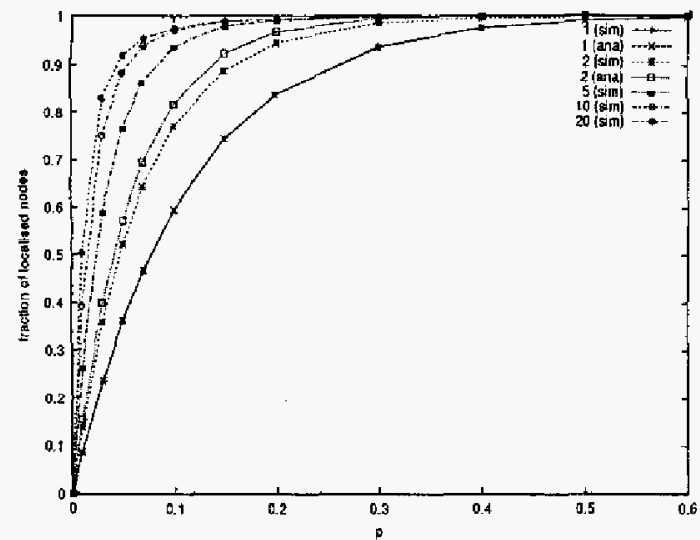

Fig. 4. Fraction of localised nodes vs. $p$ for $\lambda=2$ per $m$, $R_{0}=2 \mathrm{~m}$. Analysis and Simulation.

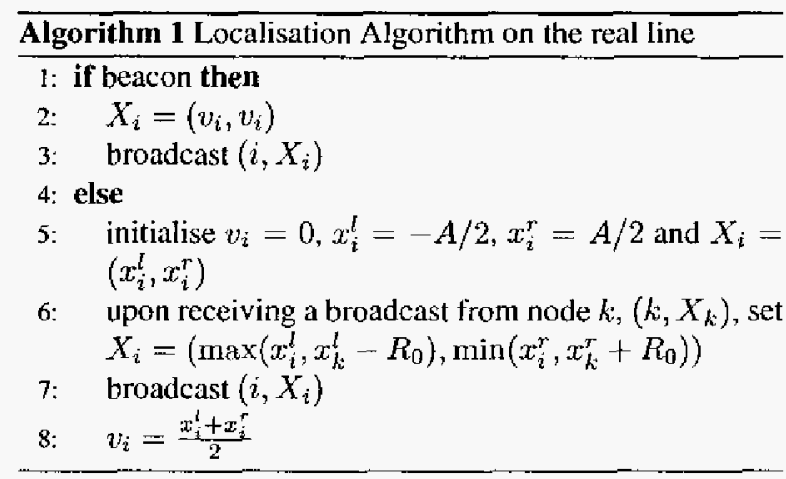

does not depend on $\lambda$ being high. Note from Proposition 4.1 that for a given $p$, localisation errors will be small if $\rho:=$ $\lambda 2 R_{0}$ is large. Similarly, the fraction of sensors which get localised per iteration will be large for large $\rho$ as Proposition 4.3 shows. Therefore, even in networks which are not very dense, IR will be an effective method as long as $\rho$ is large.

\section{LOCALISATTON ALGORITHM}

The iterative scheme in (3-4) is easy to implement in sensor networks as a distributed asynchronous algorithm 1 . In Algorithm 1 each sensor positions itself to the center of its localisation set. Figure 5 shows the variation of location estimation error (normalised to range $R_{0}$ ) with $p$ using $\mathrm{Al}$ gorithm 1 for increasing iterations. $\lambda=2$ perm and $R_{0}=2$ m. Observe that with only $15-20 \%$ beacons, sensors are able to estimate their locations with an average estimation error less than $30-35 \%$ of the range. By choosing parameter $p$ appropriately accuracy can be traded with the cost according to the needs of the application.

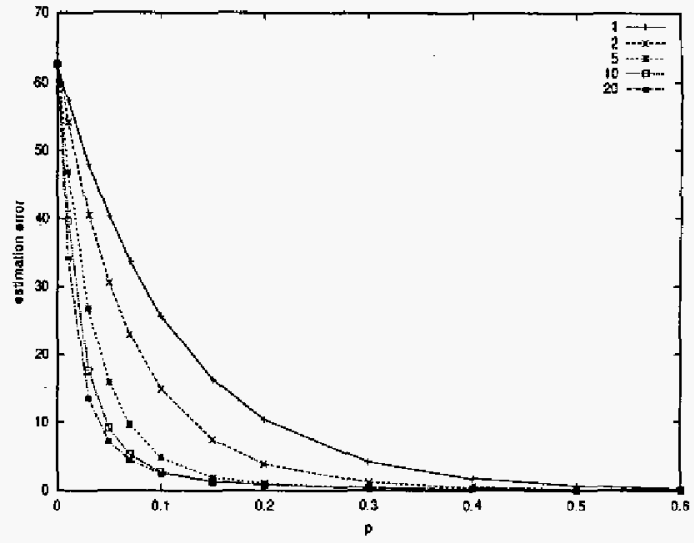

Fig. 5. Location estimation error (nornalised to $R_{0}$ ) vs. $p$ for $\lambda=2$ per $\mathrm{m}, R_{0}=2 \mathrm{~m}$ using Algorithm 1 .

\section{CONCLUSION}

We proposed an extremely simple method of localisation that relies only on the basic communication capability of sensors. The method is rendered efficient because a sensor, after localising itself, aids others in localisation. We analysed the localisation process on the line and gave an iterative method of computing the average localisation error and the fraction of sensors localised. The results show that if the number of neighbours of sensors are sufficiently high, the sensors can localise themselves collaboratively with small error using only a few beacons among them.

\section{REFERENCES}

[1] A. Savvides et al. Dynamic Fine-Grained Localization in Ad Hoc Networks of Sensors. In ACM SIGMOBILE, 2001 .

[2] R. Nagpal et al. Organising a Global Coordinate System from Local Information on an Ad Hoc Sensor Networks. In 2nd Intl. Workshop on Information Processing in Sensor Networks (IPSN), 2003.

[3] J. Gibson. The Mobile Communications Handbook. IEEE Press, 1999.

[4] A. Kamik. Optimal Self-organisation of Wireless Sensor Networks. PhD thesis, Indian Institute of Science, 2004.

[5] D. Niculescu and B. Nath. Ad Hoc Positioning System (APS). In IEEE INFOCOM, 2003.

[6] S. Simic and S. Sastry. Distributed Localization in Wireless Ad Hoc Networks. Memo. No. UCB/ERL $\mathrm{M} 02 / 26,2002$. 